\title{
Valoración coste eficacia de las transacciones de agua inter cuencas. Una aplicación a las cuencas mediterráneas andaluzas
}

\author{
Sofía OlMeDIlla Pérez \\ Ministerio de Medioambiente rural y Marino \\ sofiolme@gmail.com \\ almudena GóMez -r aMos \\ Universidad de Valladolid \\ almgomez@iaf.uva.es
}

recibido: 5 de Octubre de 2011

enviado a evaluar: 6 de Octubre de 2011

aceptado: 13 de Octubre de 2011

\begin{abstract}
RESUMEN
e 1 objetivo de este trabajo será valorar mediante el análisis coste eficacia las cesiones de derechos de uso de agua que se han desarrollado en el marco de la actual ley de aguas como medidas básicas que complementan las que actualmente propone el Plan Hidrológico de las Cuencas Mediterráneas a ndaluzas. Dichas cesiones han sido desarrolladas para mejorar el estado ambiental de las masas de agua correspondientes a la cuenca baja del río a lmanzora. e 1 análisis se desarrolla teniendo presente la incertidumbre en la disponibilidad de los recursos a partir de la consideración de distintos escenarios hídricos. 1 os resultados muestran que las cesiones de aguas tienen, desde el punto de vista del análisis coste-eficacia, una valoración más favorable que las medidas consideradas explícitamente en el Plan de las cuencas. el estudio además incorpora una reflexión sobre la idoneidad de este tipo de análisis frente a la consideración de otras variables que permitan considerar además/a parte de los costes económicos, los costes sociales y ambientales que conlleva la aplicación de dichas medidas.
\end{abstract}

Palabras clave: análisis coste eficacia, transacciones de derechos de agua, trasvase, masa de agua, acuífero, Directiva Marco del agua

\section{Cost-effectiveness assessment of transactions internationalwater basins. a $\mathrm{n}$ application to the a ndalusian Mediterranean basins}

\begin{abstract}
The purpose of this study is to evaluate through a cost-effectiveness approach water use transactions implemented under Water law framework. These measures complement others proposed by Water Plan of the Mediterranian-andallucian Basin. This water transfers were planned in order to improve the environmental conditions of the water body belonging to low Basin of almanzora river. The analysis
\end{abstract}


was developed including uncertainty related with the availability of resources under various water scarcity scenarios. The results show that water use transactions', from a cost effectiveness approach, has a more favorable assessment than the measures which are explicitly outlined in the river Basin Plan. Furthermore, the study also incorporates a reflection about the suitability of this approach in front of others that consider not just the economic costs, but also others variables, such as social and environmental cost concerned in implementing such measures.

Keywords: Cost-effectiveness analysis, water rights transactions, transfer, body of water, aquifer, Water Framework Directive

\section{RÉSUMÉ}

1 'objectif de cette étude est d'évaluer la rentabilité de la cession des droits d'utilisation de l'eau, développé sous la loi actuelle de l'eau. Ces sont mesures de base qui complètent celles qui sont actuellement proposées dans le Plan Hydrologique du Bassin Méditerranéen a ndalou, et ces sont développés pour améliorer l'état environnemental des plans d'eau pour le bassin inférieur du fleuve a lmanzora. 1 'analyse est développée en tenant compte de l'incertitude quant à la disponibilité des ressources, de l'examen des scénarios d'eau différents. 1 es résultats montrent que les transferts d'eau avent une mieux relation coût-efficacité que celles qui sont explicitement dans le plan du bassin. 1 'étude comprend aussi une réflexion sur l'adéquation de cet type d'analyse à la contrepartie d'autres variables à considérer en plus les coûts économiques, comment les coûts sociaux et environnementaux impliqués dans la mise en œuvre de telles mesures.

Mots clé: analyse coût-efficacité, les droits de transaction d'eau, le transfert, le corps de l'eau, aquifère, la directive-cadre sur l'eau

\section{INTRODUCCIÓN}

la aplicación de los principios que encierra la Directiva Marco del agua (Directiva 2000/60 Ce, DMa en adelante) a los Planes Hidrológicos de Cuenca se encuentra en proceso de adaptación. Diciembre de 2009 fue el plazo establecido desde la Ue para la aprobación de dichos planes tras el necesario período destinado a la participación pública. Sin embargo la práctica totalidad de proyectos de Planificación Hidrológica de cuencas está sufriendo un retraso y ninguno de ellos ha sido aprobado en la fecha prevista. este período de tiempo hasta la aprobación definitiva de los Planes, está permitiendo profundizar en aspectos fundamentales de la DMa que no han podido ser abordados con suficiente rigor en los mismos. la idea de los planificadores es ir incorporando en el período de revisión y seguimiento de los Planes, fase que se inicia una vez ya han sido aprobados, los avances habidos en aquellos aspectos que no han sido tratados con suficiente rigor. en concreto, los temas abordados de forma más superficial son aquellos ligados con los aspectos económicos de la DMa como puedan ser el análisis de la recuperación de los costes ligados al uso del agua o la valoración de las medidas para la recuperación ambiental de las masas de agua. este último tema es de los de mayor trascendencia y no ha sido tratado con la preferencia que la DMa exige. en este proceso de revisión se pretende incorporar aspectos como es la concreción de metodologías para el análisis y valoración de las medidas propuestas para alcanzar los objetivos ambientales de las masas de agua dentro del propio horizonte temporal de cada una. 
el trabajo que aquí se expone se ubica en este contexto. en concreto trata de profundizar en aspectos ligados con la aplicación de la metodología del análisis de los programas de medidas destinados a la recuperación ambiental de las masas de agua tal y como se entiende desde la DMa, reflexionando sobre aspectos relativos a la valoración de los costes y beneficios económicos, sociales y ambientales que dichas medidas implican revisando aspectos metodológicos y limitaciones de éstos.

este trabajo se centra en el análisis de medidas básicas (consideradas así por no requerir inversión pero que su aplicación esté intrínseca en la normativa vigente), y que no han sido incluidas en los planes de cuenca. Se trata en este caso de las cesiones temporales de derechos de uso de agua que recoge nuestro ordenamiento jurídico en materia de aguas. así, el objetivo de este trabajo es doble: por un lado se pretende analizar el método de valoración de las medidas basado en el análisis coste eficacia ( $\mathrm{aCe}$ en adelante), que es él que a la postre la DMa recomienda con ciertas limitaciones, y por otro desde el aCe valorar las cesiones temporales de derechos de uso de agua que no han sido consideradas en los Planes en proceso de aprobación. en definitiva se pretende realizar nuevas aportaciones desde el ámbito académico, en el actual proceso de incorporación de los aspectos económicos de la DMa a la planificación de las cuencas españolas, en lo que se refiere a la valoración económica de las medidas para la recuperación ambiental de una masa de agua.

Para realizar este análisis se ha recurrido a un caso real muy significativo pues se trata de una masa de agua muy modificada como es el acuífero 06.06 Bajo a lmanzora perteneciente a las Cuencas Mediterráneas a ndaluzas, que en la actualidad se encuentra totalmente sobreexplotado y donde los niveles de concentración de nitratos superan con creces los niveles mínimos que establece la Directiva de Nitratos. e ste trabajo pretende no sólo presentar una aplicación empírica del método a Ce a una caso concreto de una masa muy modificada incluyendo medidas novedosas como es las cesiones temporales de derechos de uso de agua, sino también reflexionar sobre las limitaciones del método a Ce en cuanto a la no inclusión de aspectos relevantes como pueda ser la valoración de los costes y beneficios sociales y ambientales ligados a la implementación del programa de medidas que se propone.

el trabajo se ha organizado del siguiente modo: en el siguiente punto se reflexiona sobre los aspectos económicos de la DMa y los programas de medidas, su inclusión en los Planes de cuenca y el papel que juegan en la consecución de los objetivos ambientales a través de una revisión de la literatura al respecto. en el tercer punto se profundizará en la metodología establecida por la DMa para valorar el programa de medidas. el cuarto punto se destina a presentar la problemática de la zona de estudio que abarca la zona de influencia del acuífero 06.06 y las medidas que este trabajo propone para la recuperación cuantitativa de dicho acuífero y que se centra en la cesión temporal de derechos de uso. el quinto punto presenta los resultados obtenidos en la aplicación del aCe para cada medida. Por último, se avanzan las principales conclusiones que se extraen de este estudio y que son de interés para el actual proceso de planificación.

en ningún caso este trabajo pretende proponer medidas para su posible incorporación en los planes de cuenca, sino que lo que se persigue es hacer un ejercicio de 
prospección de posibles efectos en la recuperación de la masa de agua objeto de este estudio, así como valorar las implicaciones en el área de influencia de la zona regable incluyendo las consideraciones oportunas sobre los efectos ambientales de la continuidad del escenario tendencial.

\section{EL MARCO DE LA DMA Y LOS OBJETIVOS AMBIENTALES}

el objetivo de la Directiva Marco del agua es conseguir el buen estado de las masas de agua en la Ue en el año 2015. establece que es preciso prevenir el deterioro adicional de ecosistemas acuáticos, promover el uso sostenible del agua, incrementar la protección del medio acuático y la reducción progresiva de la contaminación del agua subterránea. De este modo, los objetivos ambientales pasan a ser una condición previa, y por lo tanto primordial, a los objetivos de atención de demandas -solicitudes de volúmenes de agua por parte de actividades socio-económicas(Maeztu y Domingo, 2008).

el nuevo proceso de planificación hidrológica promovido por la DMa refuerza la necesidad de alcanzar dichos objetivos ambientales para cada masa de agua a través del diseño y valoración de un programa de medidas. la normativa española exige que los organismos de cuenca elaboren una lista de objetivos ambientales para cada una de las masas de agua, incluyendo los plazos previstos para su consecución. Para las aguas subterráneas, que son las que se valoran en este trabajo, los objetivos ambientales establecidos por la DMa implican un buen estado cuantitativo (equilibrio entre las extracciones y las recargas) y una mejora del estado químico (prevención o limitación de la contaminación e inversión de esta) siempre bajo el principio de no deterioro respecto al estado actual. Dependiendo del estado actual de la masa de agua se establecerá la necesidad o no de tomar medidas para la consecución de tales objetivos. el objetivo de las mismas es por tanto, estrechar la 'brecha' entre el estado actual o tendencial de las masas de agua y el estado objetivo previamente establecido (Berbel et al., 2009).

la caracterización del uso del agua en las diferentes demarcaciones pone de manifiesto que las principales presiones que afectan a las masas de agua tienen un carácter tanto cuantitativo como cualitativo, proveniente en la mayor parte de los casos por un uso intensivo del recurso. esta situación es especialmente patente en las masas de agua que son fuente de suministro de agua de riego en las zonas donde la agricultura es más intensiva en el uso de inputs (Sauquillo et al., 2009; Garrido et al., 2006, almasri, 2007). la revisión de las medidas propuestas para la recuperación de las masas de agua subterráneas incluidas en los Planes Hidrológicos de las demarcaciones en las que éstos problemas son más evidentes, y por tanto se encuentran más deterioradas, pone de manifiesto que existe una mayor preocupación del organismo planificador por la recuperación cuantitativa de estas masas a través del equilibrio entre extracciones y recargas, frente a la recuperación cualitativa de las mismas. De este modo, los objetivos de prevención adicional del deterioro de los ecosistemas, el uso sostenible de los recursos o la reducción progresiva de la contaminación, dejan 
de ser objetivos prioritarios y pasan a ser considerados como exenciones u objetivos menos rigurosos (Gómez-ramos y Olmedilla, 2011).

la DMa, aunque considera fundamental los aspectos económicos en la gestión del agua, no establece una primacía de este enfoque en la toma de decisión. Por el contrario, la DMa considera a la economía como una herramienta que debería estar subordinada a la consecución del objetivo ecológico (Ferrer y la roca, 2006; Gómez, 2006). Sin embargo, la DMa propone el análisis coste-eficacia como método apropiado para la selección de medidas más adecuadas para alcanzar los objetivos ambientales. a través de la Guía Wateco (CIS, 2003) la DMa establece que es necesario analizar inicialmente cada una de las medidas que potencialmente pueden emplearse para alcanzar los objetivos ambientales. Posteriormente, esta primera selección de actuaciones debe caracterizarse pormenorizadamente en cuanto a sus consecuencias socioeconómicas (costes asociados) y ambientales (eficacia en la consecución de objetivos), al objeto de proporcionar una herramienta de evaluación estratégica que ayude a seleccionar del conjunto de medidas que resulten más eficaces y eficientes a un mínimo coste. Si de este análisis se evidenciase que los costes necesarios para alcanzar los objetivos ambientales en determinadas masas de agua son excesivamente elevados ("costes desproporcionados" empleando el lenguaje de la DMa), tal circunstancia permitirá justificar una exención en relación al logro del buen estado, pudiéndose plantear para dichas masas un aplazamiento para el logro del buen estado o un objetivo menos riguroso. las excepciones posibles en los objetivos de la DMa pueden considerarse de dos tipos: que los costes sean desproporcionados respecto a la capacidad financiera para asumirlos, o que los costes del plan sean desproporcionados respecto a los beneficios que supone la mejora en la calidad ambiental. el primer caso, se trata de un claro problema de capacidad financiera que depende del modo en que se repercutan los costes del Plan de Cuenca sobre los usos del agua, y que sólo justificaría el aplazamiento de los objetivos ambientales (Gómez, 2006). en el segundo caso, la desproporción es relativa a la bondad de las ventajas de los planes de cuenca en relación con sus costes y puede conllevar a una posible rebaja de los objetivos del buen estado. Mientras que en el caso anterior los mecanismos financieros pueden ser suficientes para la derogación temporal de los mismos, en el caso de la rebaja de los objetivos será necesario realizar un análisis coste-beneficio en el que se incluyan los costes y beneficios sociales y ambientales completos asociados con las medidas (Martín- Ortega y Berbel 2008).

a la hora de establecer las medidas es preciso considerar y valorar las medidas básicas que no requieren inversión y por tanto no implican costes adicionales. estas medidas suponen la aplicación real de toda la legislación en materia ambiental que indudablemente mejorará el estado ambiental de la masa de agua. el método aCe tal y como se presenta en la guía Wateco, presenta importantes limitaciones en cuanto a la valoración real de los costes y beneficios ligados a las medidas puesto que no toma en consideración los beneficios que la sociedad obtiene del buen estado ecológico de las masas de agua (externalidades positivas) y el hecho de que los costes ambientales se correspondan con los "beneficios perdidos" (coste de oportunidad) de no alcanzar dicho estado. además sería necesario considerar el coste del recurso como 
el coste de oportunidad de utilizar el recurso escaso agua para un determinado uso más allá de la capacidad natural de recarga o recuperación. el método aCe tal y como establece la guía Wateco, se limita a valorar las medidas de reparación, mitigación y prevención derivadas de la provisión del recurso (Brouwer y Georgiou, 2007) pero no tienen en cuenta cuál es el valor del bien medido como aquel que la sociedad confiere a la disponibilidad del recursos en un estado adecuado y que se mide por su disposición a pagar $(\mathrm{DaO})$ y a aceptar (Daa). es el análisis coste beneficio el que permite aproximar mejor este valor.

la dificultad de este método estriba en la estimación de los beneficios ambientales pues tiene limitaciones en cuanto a las metodologías disponibles para tal fin, ya que exigen un profundo trabajo de campo que debe ser muy exhaustivo para alcanzar resultados contrastables y realmente rigurosos. ello encarece los trabajos y los hace prácticamente inabordables. es por ello que la DMa, bajo un enfoque pragmático, recomienda el aCe como metodología a seguir. No obstante desde el ámbito académico se han realizado algunas aplicaciones empíricas que tratan de incorporar los métodos propios de la economía ambiental para valorar los beneficios ambientales. Del Sanz, 2009 y Martín Ortega, 2008 han aplicado estos métodos en la valoración coste-beneficio de algunas medidas ambientales en el marco de la DMa para la cuenca del rio Serpis en el primer caso y para la cuenca del Guadalquivir en el segundo. No obstante, desde el punto de vista de la economía ecológica (otra corriente de pensamiento en economía de los recursos naturales) este enfoque tiene también detractores pues subvierte la lógica de la DMa, ya que la monetización de los costes y beneficios ambientales supone la consecución o revisión de los objetivos ambientales a la aplicación de una regla de decisión económica (la roca, 2003), que contraviene como se dijo anteriormente los principios de la DMa.

en lo que se refiere a la aplicación empírica del aCe al caso de la recuperación cuantitativa de una masa de agua son varios los trabajos realizados hasta la fecha, centrados en su mayoría en países mediterráneos pues es aquí donde los problemas en la cantidad del recurso son más acuciantes. en esta línea cabe destacar el trabajo pionero realizado en la Cuenca del rio Cidacos por el Ministerio de Medioambiente y el Gobierno de Navarra en el año 2002. en dicho forme se hace un repaso cualitativo de los beneficios ambientales potenciales asociados a las medidas de mejora de la calidad de las aguas: beneficios relacionados con las actividades recreativas, con las actividades productivas y finalmente relacionados con la conservación o restauración de los servicios asociados con al ecosistema fluvial. Por su parte, los trabajos de Álvarez-Farizo y Hanley (2006) y Álvarez-Farizo et al. (2006) tienen su origen en el proceso de consulta y participación pública desarrollados durante la elaboración del mencionado Plan de Cuenca del río Cidacos.

Otro trabajo de referencia en el análisis coste-eficacia es el que se centra en la subcuenca del Jalón (Confederación Hidrográfica ebro-MIMaM, 2008). en este trabajo se recoge la valoración económica de de las distintas obras hidráulicas (revestimiento de acequias, balsas de almacenamiento, etc.) en relación con la mejora de la calidad de las aguas (reducción del nitrógeno vertido). en este trabajo como novedad también se valoran medidas de carácter básico como pueda ser el asesoramien- 
to al regante, código de buenas prácticas y riego deficitario que no vienen recogidas en las medidas con impacto cuantitativo. a todos los trabajos anteriormente citados hay que añadir los que están actualmente representados en el proyecto de investigación europeo aquaMoney (2010) que tiene como objetivo el desarrollo de guías prácticas para la valoración económica de los beneficios y costes ambientales en el contexto de la DMa.

\section{METODOLOGÍA}

1 a DMa , como se ha mencionado en el apartado anterior, presionada por la urgencia de las decisiones y la falta de información, adoptó soluciones prácticas muy reduccionistas a la hora de abordar la valoración económica de las medidas necesarias para alcanzar los objetivos ambientales previstos para la masa de agua. a sí, propuso el a Ce como método general y en casos excepcionales el análisis coste-beneficio (a CB en adelante) ver guía Wateco (Comisión e uropea, 2003)-. el enfoque a Ce se basa en comparar la reducción del impacto sobre el medio acuático (del parámetro en cuestión) versus coste anual equivalente (financiero) de la medida. Por el contrario, el enfoque a CB se propone como instrumento para tratar la posible derogación o prórroga de los objetivos ambientales. 1 a DMa propone de este modo, el uso del análisis coste-eficacia de medidas e impactos como propuesta para evitar la 'monetarización' y complejidades del a CB (Berbel, 2009). Con el a Ce se genera un ranking de medidas ordenadas por el ratio coste anual equivalente/reducción de impactos (o presiones). Desde el punto de vista metodológico, esto supone una cierta ordenación lexicográfica donde se pretende minimizar el coste financiero una vez que se alcanza la meta ambiental fijada para cada masa de agua (Kranz et al., 2004). No obstante este enfoque lexicográfico impide el análisis de 'tradeoffs' entre objetivos ambientales y sociales, lo que enriquecería el análisis y mejora el proceso de decisión y de participación pública (Martínez-a lier et al, 1998).

el aCe supone calcular el índice coste-eficacia, que será el cociente entre el coste anual equivalente y la mejora efectuada con esa medida. Para cada aspecto que se pretenda conseguir se ordenarán de menor a mayor las medidas propuestas en función del índice coste-eficacia obtenido, y se seleccionarán aquellas medidas con menor índice coste-eficacia que sean suficientes para conseguir el objetivo fijado. es necesario tener una descripción de las características de la medida que se quiere evaluar, indicando en qué va a consistir esa medida y qué finalidad se va conseguir con su aplicación. También hay que especificar si alguna medida requiere de la aplicación de otra para su consecución, además de si la medida tiene un carácter básico o complementario, entendiendo por las primeras aquellas que no requieren inversión y que son consecuencia de la aplicación de la norma correspondiente, mientras que las segundas implican la puesta en marcha de nuevas infraestructuras o la aplicación de nuevos instrumentos o mecanismo no contemplados hasta el momento. en su caso, se detallarán los elementos que componen la medida. además hay que indicar a qué tipo de indicador afectará la medida (indicadores biológicos, hidromorfológicos o fisicoquímicos si hablamos de aguas superficiales, e indicadores del estado químico 
o cuantitativo para el caso de aguas subterráneas). De este modo es posible identificar las presiones que son eliminadas o mitigadas si se aplican las medidas.

el coste en el aCe se refiere al "coste directo de la medida, que incluye costes de inversión anualizados, coste de mantenimiento y otros costes económicos que implique la medida". Para aquellas medidas que se haya hecho la cuantificación del coste, su valoración deberá ser común en toda la demarcación hidrográfica para evitar la introducción de sesgos y deberá ser posible realizar el análisis coste-eficacia en el que se apoyará la selección de la combinación de medidas más adecuada para cumplir con los objetivos propuestos. el coste de las medidas se expresará como Coste anual equivalente ( $\mathrm{Cae}$ en adelante), excluidos los impuestos, e incluyendo los costes directos e indirectos:

Para el cálculo del Coste anual equivalente, se seguirá la siguiente fórmula:

$$
\text { C.A.E. }=I \bullet a_{n, i}+\text { C.E.M. }
$$

Donde $I$ es la inversión requerida, $a_{n, i}$, es el factor de amortización anual. y $C M$ es el coste de explotación y mantenimiento. También se considerarán los costes económicos, sociales y ambientales -los costes indirectos-, integrándolos en el coste anual equivalente cuando sea posible su cuantificación en términos monetarios. en el cálculo de la anualidad deberá tenerse en cuenta, en su caso, la vida útil de todos y cada uno de los elementos necesarios para la ejecución de la medida, el horizonte temporal para el que se realiza el análisis y el plazo de ejecución de la medida hasta su puesta en marcha. Deberá especificarse la tasa de descuento utilizada para el cálculo de la anualidad. el coste de las medidas se valorará a precios constantes indicándose el año de referencia utilizado.

Para evaluar la eficacia de las medidas se partirá de la evaluación del estado de las masas de agua correspondiente al escenario tendencial sin aplicar las medidas propuestas y se calculará la diferencia respecto al estado ambiental de las masas de agua con la aplicación de las medidas. 1 a diferencia que exista entre ambos estados será el grado de eficacia de la medida. Por lo tanto, la eficacia de una medida se define como la aportación que ésta hace a la consecución de los objetivos de estado en una o varias masas de agua. Puede evaluarse de dos formas: mediante la reducción de las presiones significativas que sufren las masas de agua, es decir, reducción de la magnitud de las presiones reflejadas en el inventario de presiones o mediante la reducción de los impactos medidos en las masas de agua, es decir, mejoras en los indicadores de estado de las masas de agua que propicia la medida. e ste método de evaluación requiere conocer previamente la eficacia de la medida en la reducción de presiones y la susceptibilidad de las masas de agua ante estas presiones. 1 a eficacia se evaluará preferentemente como una reducción de impactos, siendo la primera una alternativa válida ante limitaciones de información sobre la susceptibilidad de las masas de agua Para Berbel (2008) es fundamental considerar el grado de incertidumbre en la consecución de los objetivos s la hora de valorar la eficacia. Para incorporar este elemento en la estimación de la eficacia dado que esta depende de condicionantes climáticos se recurrirá a su expresión a través de un rango de valores. 


\section{CASO DE ESTUDIO}

el uso intensivo de las aguas subterráneas en las zonas áridas y semiáridas para el riego ha experimentado un espectacular crecimiento en los últimos cuarenta años en el levante español. llamas y Martínez-Santos (2005) han denominado a este efecto como la revolución silenciosa pues ha sido llevada acabo por pequeños y medianos agricultores en un contexto de práctica ausencia de control y planificación por parte de las autoridades de cuencas encargadas de su gestión. Por otra parte el riego intensivo con aguas subterráneas ha generado importantes benéficos sociales y ambientales en el área de influencia (Sánchez Picón et al. 2011), lo que ha supuesto paralelamente, y en buena medida agudizada por la falta de gobernanza, la degradación de las masas de agua no sólo por la sobre explotación de los acuíferos sino por el aumento progresivo de la concentración de contaminantes originado por la contaminación difusa en dichas aguas, además de otros efectos ambientales ligados con la generación de residuos sólidos, cambios en el paisaje, presencia de plaguicidas en suelos, plásticos y agua (Tolón y lastra, 2010).

la alta productividad del agua destinada al riego de estas áreas estimada en 4 $€ / \mathrm{m}^{3}$ (1lamas y Garrido 2007; Palancar, 2000) junto con las altas tasas de empleo generado (300 Jornales por días y hectárea para cultivos como el tomate o el pimiento) conviven con el desarrollo reciente de otras actividades como el turismo o la construcción (Cortés et al.2002). la continúa generación de riqueza en la zona, frenada en los tres últimos años por la crisis del turismo y construcción, han justificado la continuidad del modelo productivo. Sin embargo, en los últimos años, y en buena medida debido a las mayores exigencias ambientales del consumidor que es transmitida por la gran distribución, existe una reorientación de la producción hacía un modelo de producción integrada e incluso ecológica (llamas y Garrido 2007). en cualquier caso son muchas las voces que siguen cuestionando la sostenibilidad social y ambiental de este modelo de producción (estevan y Naredo 2004; arrojo, 2001).

Para este trabajo se propone aplicar el aCe al programa de medidas propuesto para alcanzar los objetivos ambientales ligados con los valores cuantitativos de la masa de agua subterránea Bajo almanzora (06.06), que suministra agua de riego a la zona regable Cuevas de almanzora, que reúne la esencia de la agricultura intensiva antes descrita. es por ello que la masa se puede definir como muy modificada pues según datos recientes está sobreexplotada en una cantidad de $0,28 \mathrm{Hm}^{3} /$ año (se extraen $2,20 \mathrm{Hm}^{3}$ para unas disponibilidades de $1,93 \mathrm{Hm}^{3}$ ). la tendencia, si no se aplican medidas adicionales tal y como establece el Plan de Cuenca para el año 2015, es que se agrave la situación aumentando las extracciones a $2,5 \mathrm{Hm}^{3}$, estimando una sobreexplotación de $0,58 \mathrm{Hm}^{3}$ /año (Plan Hidrológico de las Cuencas Mediterráneas andaluzas. agencia andaluza del agua).

el origen de la sobre explotación de la masa son los usos agrarios. la masa abastece 19.394 has de regadío destinado al cultivo de hortícolas al aire libre (40\% de la superficie) y cítricos (60\% de la superficie) regados mayoritariamente con riego por goteo. la demanda actual para riego se estima en $62 \mathrm{Hm}^{3}$ al año, que equivalen a 
unos $3.248 \mathrm{~m}^{3} /$ ha. la procedencia de los recursos para abastecer esta demanda se presenta en el cuadro 1 .

Cuadro 1: Balance Hídricos de la zona regable que abastece la masa de agua Bajo almanzora

\begin{tabular}{|c|c|c|}
\hline \multirow{2}{*}{ NECESIDADES HÍDRICAS } & \multicolumn{2}{|c|}{ APORTACIONES HÍDRICAS } \\
\hline $62.981 .968 \mathrm{~m}^{3}$ & SUBTerrÁNea & $7 \mathrm{Hm}^{3} \rightarrow$ Concesiones \\
\hline \multirow{3}{*}{} & & $8 \mathrm{Hm}^{3} \rightarrow$ Sobreexplotación \\
\cline { 2 - 3 } & & TOTAL: $\mathbf{1 5 . 0 0 0 . 0 0 0} \mathbf{~ m}^{\mathbf{3}}$ \\
\cline { 2 - 3 } & SUPerFICIal & TOTAL: $\mathbf{4 7 . 9 8 1 . 9 6 8} \mathbf{m}^{\mathbf{3}}$ \\
\hline
\end{tabular}

Fuente: elaboración propia con datos de aCUaMeD (2006) y de los regante de la zona

1 a procedencia del agua para riego en la zona regable de este estudio es diversa, teniendo poco peso el agua de origen superficial procedente de cursos fluviales, mientras que ha adquirido gran importancia en los últimos años el agua procedente del trasvase del embalse del Negratín (Sanchez Picón et al, 2009). 1 as aguas subterráneas aportan $15 \mathrm{Hm}^{3}$ de estos recursos. De esto $8 \mathrm{Hm}^{3}$ proceden de pozos salinos pues las Comunidades de $r$ egantes de la zona pusieron en marcha una pequeña desaladora de agua salobre para riego. 1 os $7 \mathrm{Hm}^{3}$ restantes proceden de acuíferos, los cuales se encuentran en una situación de infradotación y sobreexplotación declarada como ya se ha visto. e l déficit hídrico estimado para la zona es de $15 \mathrm{Hm}^{3}$. e sta situación de sobre explotación de acuífero implica una fuerte presión de tipo cualitativo sobre éste con una concentración de nitratos de 205mg/1 que supera con creces los $50 \mathrm{mg} / 1$ establecidos en la Directiva de Nitratos. a demás de estos, se están superando los valores umbrales de otros contaminantes como amonio, cloruros o sulfatos.

Desde su entrada en funcionamiento en el año 2005, el Trasvase del Negratín es la principal fuente de recursos hídricos de la comarca. é ste se pone en marcha por iniciativa de los propios regantes y usuarios de aguas de la zona del levante almeriense ante la falta de recursos. Dicho trasvase se rige por la ley del trasvase 1 ey 55/1999, que dispone un máximo a trasvasar de $50 \mathrm{Hm}^{3}$ de aguas excedentarias entre la cuenca del $\mathrm{r}$ ío Guadalquivir, más concretamente la cuenca del Guadiana Menor y la cuenca mediterránea andaluza, en la cuenca del $r$ ió a lmanzora. 1 a concesión de este trasvase es de la empresa a guas de a lmanzora que representa a la Junta Central de Usuarios del Valle de a lmanzora. 1 a condiciones del trasvase que regula la ley 62/2003 se establecen en base a las disponibilidades de la cuenca cedente y son las siguientes: se trasvasará sólo si el volumen excede de $210 \mathrm{Hm}^{3}$ en el embalse cedente y sólo cuando el volumen embalsado en el Sistema de explotación de r egulación General supere el mínimo del 30\% de la capacidad de embalse. Se trata por tanto de una medida de tipo básico que si bien es cierto, aporta una buena cantidad de recursos hídricos a la zona, cuenta también con una elevada incertidumbre ligada al cumplimiento de las condiciones antes citadas. Para estimar las disponibilidades hídricas reales procedentes del trasvase se ha procedido al cálculo de probabilidades del cumplimiento de las condiciones para poder trasvasar, atendiendo a los datos históricos sobre el nivel de reservas del embalse del Negratín. Se han supuesto tres escenarios posibles: que se cumplan las condiciones para trasvasar agua, que no se 
cumplan por no alcanzarse los $250 \mathrm{Hm}^{3}$ exigidos y por último que se produzca una situación de llenado total en el embalse que provoca aliviados y por tanto, un desembalse extra debido a la incapacidad de éste de recoger todas las aportaciones recibidas. Para estas situaciones se acordó con la Junta Central de Usuarios del Valle de a lmanzora el no cobrar el Canon de regulación a los usuarios ya que sería una decisión que beneficiaría a ambos al evitar las avenidas en el Guadalquivir. e n el cuadro 2 quedan recogidas las probabilidades que se han calculado a partir de los datos de los niveles de los embalses del Sistema de explotación General al cual pertenece el embalse del Negratín.

Cuadro 2: Probabilidad de que se produzca el trasvase según la situación de la cuenca cedente

\begin{tabular}{|l|c|}
\hline SITUACIÓN DE TRASVASE & PROBABILIDAD (\%) \\
\hline Que se realice el Trasvase (año NOrMal) & $54,67 \%$ \\
\hline Que no se realice el Trasvase (año SeCO) & $44 \%$ \\
\hline Que el trasvase se realice sin pago canon & $1,33 \%$ \\
\hline TOTAL & $100 \%$ \\
\hline
\end{tabular}

Fuente: elaboración propia a partir de los datos de la agencia andaluza del agua (2008)

e n este contexto de incertidumbre ligado al trasvase, se han implementado en los últimos años cesiones temporales de derechos de uso de carácter voluntario. Debido al bajo nivel de las reservas en la cuenca del Guadalquivir, durante las campañas de riego 2006-2007 y 2008 fue necesario tomar medidas restrictivas en las dotaciones de consumo agrícola, y por tanto una vez declarado la situación de excepcionalidad que establece la 1 ey de aguas, se autorizaron cesiones temporales entre usuarios (Calatrava y Gómez $\mathrm{r}$ amos, 2009). en este contexto legal, el MMa autorizó en 2006 un contrato de cesión temporal de derechos hídricos entre regantes pertenecientes a la cuenca del Guadalquivir y a la Cuenca Mediterránea a ndaluza, a través de la infraestructura del Trasvase Negratín-a lmanzora. e sta operación de derechos se realizó entre los propietarios de la finca arrocera "Dehesa Norte S.a ." ubicada en la provincia de Sevilla y la entidad a guas de a lmanzora de a lmería, y con ella se iniciaron los intercambios correspondientes a la adquisición de 1.600 Has de fincas situadas en la zona arrocera de la parte baja del Guadalquivir (Sanchez Picón et al, 2009). e sta autorización permitió que se pusiera en funcionamiento de nuevo el Trasvase del Negratín-a lmanzora, que estaba paralizado desde septiembre de 2006, por haberse agotado el volumen autorizado para ese año. a simismo se produjeron cesiones de derechos, también a a guas del a lmanzora S.a ., de la C.r . del r ío Bembézar (Guadalquivir Medio) al precio 0,18 $€ / \mathrm{m} 3$ y de la C.r . del Pago de la Vega de Serón al precio de $0,15 € / \mathrm{m}^{3}$. Por la importancia que han tenido estas cesiones de derechos, en este estudio se han considerado como medidas básicas (ya que se desarrollan dentro de un marco legal preestablecido) que complementan al propio trasvase incluyéndolo de este modo en el pool de medidas posibles implementadas para contribuir a la recuperación cuantitativa del acuífero.

las medidas que se han articulado para este análisis en torno al trasvase y las cesiones de derechos se ha organizado en base a los escenarios definidos en función, por un lado del estado de la cuenca cedente, es decir, que se verifiquen las condiciones establecidas para ejercitar los trasvases y por otro, de la mayor o menor flexibilización en la autorización de cesiones de derechos, basado siempre en los precedentes antes mencionados. en el cuadro 3 se describen los 5 escenarios establecidos en función da las condiciones prefijadas. 
Cuadro 3: Caracterización de los escenarios ligados a la medida de la infraestructura del trasvase

\begin{tabular}{|c|c|c|c|c|}
\hline Escenario & $\begin{array}{c}\text { Caracterización climática } \\
\text { (probabilidad) }\end{array}$ & $\begin{array}{c}\text { Habilitación } \\
\text { cesiones }\end{array}$ & \begin{tabular}{|c|} 
Volumen \\
Trasvasado $\left(\mathbf{H m}^{3}\right)$
\end{tabular} & $\begin{array}{c}\text { Volumen cedido } \\
\left(\mathrm{Hm}^{3}\right)\end{array}$ \\
\hline e1 & Normal $(54,6 \%)$ & No & 39,6 & - \\
\hline e2 & $\operatorname{Seco}(44 \%)$ & $\mathrm{Si}$ & 12,2 & 26,8 \\
\hline e3 & $\operatorname{Seco}(44 \%)$ & No & 12,2 & - \\
\hline $\mathrm{e} 4$ & $\operatorname{Normal}(54,6 \%)$ & $\mathrm{Si}$ & 43 & 26,8 \\
\hline e5 & Húmedo $(1,4 \%)$ & No & 43 & - \\
\hline
\end{tabular}

Fuente: elaboración propia

1 as cantidades finalmente trasvasadas que aparecen en el cuadro 3 se han obtenido como media de las que ha habido en los años de análisis, considerando las probabilidades de sucesión de cada escenario. Para el caso de las cantidades cedidas a través de las cesiones de derechos, se ha tenido en cuenta la media de cesiones realizadas en los años en los que se ha implementado este mecanismo (período 2006-2009), es decir, se ha considerado una situación de estatus quo. Por tanto no se ha considerado la posibilidad de que las cesiones se incrementen en los próximos años. e n este análisis se han incluido un escenario adicional e hipotético (pues no está actualmente recogido en la normativa que regula las cesiones temporales de derechos) y que se trata de la consideración de un modelo de liberalización de la cesiones, es decir que éstas puedan producirse siempre que haya acuerdo entre las partes sin tener que ser declarada una situación de excepcionalidad hídrica tal y como sucede ahora (en el cuadro 3 coincidiría con el escenario e 4).

Por último y para finalizar con la descripción del caso de estudio, hay que mencionar la medida que explícitamente el Plan de la Cuenca Mediterránea andaluza considera para alcanzar los objetivos ambientales de la masa de agua. Se trata de la desaladora del Bajo almanzora, cuyo objetivo es generar nuevos recursos para el abastecimiento a la población y el riego en la cuenca del almanzora. la capacidad de producción de la presente desaladora es de $60.000 \mathrm{~m}^{3}$ de agua al día, que suponen unos $20 \mathrm{Hm}^{3}$ de agua al año. De estos $15 \mathrm{Hm}^{3}$ se destinarán a riego, de los que $8 \mathrm{Hm}^{3}$ servirán para paliar la sobreexplotación de los acuíferos, mientras que los 7 $\mathrm{Hm}^{3}$ restantes son para cubrir el déficit de agua y consolidar la agricultura de regadío de alto valor para la economía de la zona (aCUaMeD, 2006).

Cuadro 4. Balance Hídrico de la zona regable tras la aplicación de las medidas que establece el PHC que abastece la masa de agua Bajo almanzora

\begin{tabular}{|c|c|c|c|}
\hline \multirow{2}{*}{\begin{tabular}{|c|} 
NECESIDADES \\
HÍDRICAS
\end{tabular}} & \multirow{2}{*}{ ORIGEN DEL AGUA } & \multicolumn{2}{|c|}{ APORTACIONES HÍDRICAS } \\
\hline & & Sin medida & Con medida \\
\hline \multirow{3}{*}{$62.981 .968 \mathrm{~m}^{3}$} & \multirow{2}{*}{ SUBTerrÁNea } & $\begin{array}{l}7 \mathrm{Hm}^{3} \\
\text { Concesiones }\end{array}$ & \multirow{2}{*}{$\begin{array}{l}15.000 .000 \mathrm{~m}^{3} \\
\text { Desaladora }\end{array}$} \\
\hline & & $\begin{array}{l}8 \mathrm{Hm}^{3} \\
\text { Sobreexplotación }\end{array}$ & \\
\hline & SUPerFICIal & $\begin{array}{l}47.981 .968 \mathrm{~m}^{3} \\
\text { trasvase Tajo-Segura y pan- } \\
\text { tano de cuevas de Almanzora }\end{array}$ & $\begin{array}{l}27.620 .320 \mathrm{~m}^{3} \\
\text { Concesión Trasvase } \\
\text { Negratín } 11.798 .254 \mathrm{~m}^{3} \\
\text { Compra de derechos }\end{array}$ \\
\hline
\end{tabular}

Fuente: elaboración propia con datos de aCUaMeD (2006) y de los regante de la zona 


\section{RESULTADOS}

los resultados de este trabajo se presentan a partir de análisis de la eficacia y del coste de la combinación de medidas presentadas en el apartado anterior. a la hora de determinar la eficacia de las medidas, hay que tener en cuenta la incertidumbre asociada a la disponibilidad de determinados recursos hídricos y que ha sido ya analizada en el apartado anterior. la eficacia vendrá determinada por la reducción de las presiones procedentes de las extracciones para el riego y cuyo indicador será el agua sustitutiva procedente de las medidas antes presentadas.

Cuadro 5: Balance Hídrico en la zona de estudio con medidas

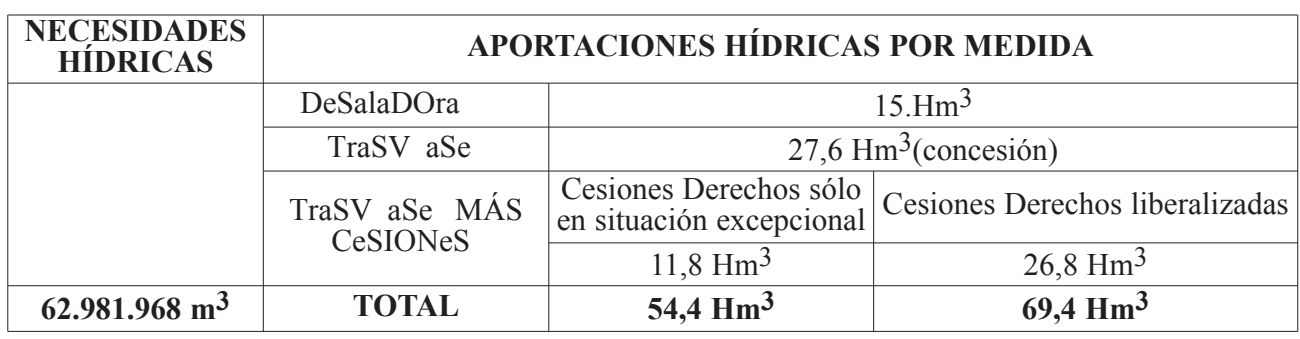

Fuente: elaboración propia

Para el cálculo del coste de las medidas se ha tenido en cuenta el coste de la inversión que implica la puesta en marcha de la desaladora y la infraestructura del trasvase más los costes de explotación y mantenimiento de éstos. Se ha estimado, para el caso del trasvase, que estos costes de mantenimiento dependerán del volumen de agua trasvasado. e n el caso de la consideración de cesiones temporales realizadas a través de las infraestructuras del trasvase, se ha tenido en cuenta el precio pagado por los regantes por el agua trasvasada que como se mencionó anteriormente fue de $0,18 \mathrm{e} / \mathrm{m}^{3}$. a demás, se han establecido los siguientes supuestos: Precios constantes, uso de la tasa de descuento del $4 \%$, que es habitualmente utilizada en proyectos hidráulicos del sector público. Duración de los convenios de explotación: 50 años para trasvases y 25 años para desaladoras. en el cuadro 6 se muestran los resultados del análisis coste eficacia para el conjunto de las medidas teniendo en cuenta la eficacia media de la medida trasvasecesión en función de la incertidumbre ligada a las condiciones hídricas que se consideran para establecer las condiciones de las cesiones de derechos (ver cuadro 3).

Cuadro 6: Índice Coste-eficacia de las medidas

\begin{tabular}{|l|c|c|c|}
\hline MEDIDA Y ESCENARIO & $\begin{array}{c}\text { COSTE ANUAL } \\
\text { EQUIVALENTE } \\
\text { CAE (€/año) }\end{array}$ & EFICACIA ( $\left.\mathbf{m}^{\mathbf{3}}\right)$ & $\begin{array}{c}\text { Índice } \\
\text { Coste-Eficacia } \\
\left(\mathbf{\epsilon} / \mathbf{m}^{\mathbf{3}}\right)\end{array}$ \\
\hline TRASVASE & $16.755 .614,67$ & 27.6 & $\mathbf{0 . 6 0}$ \\
\hline Trasvase & 19.037 .594 & 39.4 & $\mathbf{0 , 4 8}$ \\
\hline $\begin{array}{l}\text { Trasvase con Cesión } \\
\text { Derechos sólo en situación excepcional }\end{array}$ & 19.680 .840 & 54.43 & $\mathbf{0 , 3 6}$ \\
\hline $\begin{array}{l}\text { Trasvase con Cesión } \\
\text { Derechos liberalizados }\end{array}$ & 11.311 .353 & 15 & $\mathbf{0 , 7 5}$ \\
\hline DESALADORA
\end{tabular}

Fuente: elaboración propia 
el índice coste-eficacia más bajo corresponde al trasvase con Cesión de Usos de agua liberalizados, por la alta eficacia de la medida, mientras que el mayor índice es para la medida correspondiente a la puesta en funcionamiento de la desaladora. la medida del trasvase con Cesión de Usos de agua sólo en situación excepcional tiene también una mejor posición que la explotación de la desaladora, si atendemos al Índice Coste-eficacia.

en la figura 1 se muestra la curva de costes marginales asociados a incorporación de nuevos recursos al sistema con la aplicación de las diferentes medidas de forma concatenada, teniendo en cuenta el $\mathrm{Ca}$ e de cada una de las medidas consideradas. Como se observa el coste marginal mayor por $\mathrm{m}^{3}$ se debe a la puesta en marcha de la desaladora y del trasvase. 1 as medidas propuestas en este trabajo para aumentar las disponibilidades tienen un coste marginal inferior y tienen por tanto una eficacia mayor. en definitiva, el mayor esfuerzo económico se concentra en las medidas conducentes a la recuperación cuantitativa del acuífero mientras que las medidas básicas asociadas a la posible puesta en marcha de cesiones temporales tienen un coste marginal menor, entendiendo este como el coste por $\mathrm{m}^{3}$ aportado al sistema.

a la vista de estos resultados este análisis es incompleto pues parece necesario incorporar al propio coste financiero de estas cesiones, los costes indirectos tanto de carácter socio económico como ambiental que implica la trasferencia en la zona cedente, que en este caso se localizaría en las áreas de riego que contienen a las comunidades de regantes del Bembézar y del Pago de la Vega, así como a la zona productora de arroz en el Bajo Guadalquivir. e ste análisis sería objeto de otro análisis.

Figura1. Coste marginal de las medidas

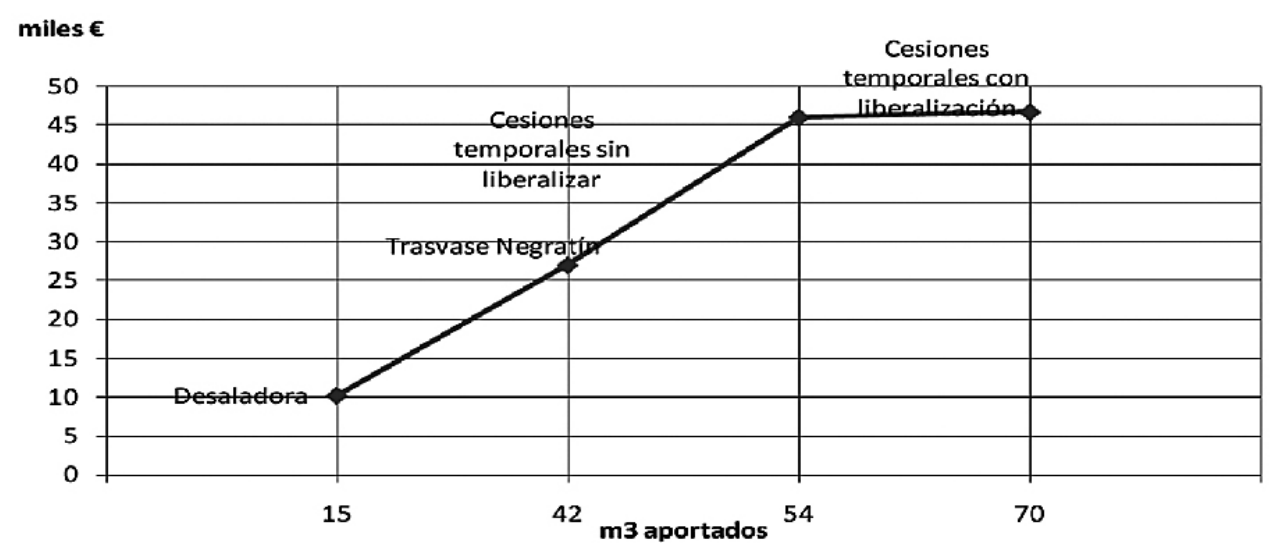

Fuente: elaboración propia 
Cuadro 7: coste asociado al uso del agua para el regante para cada una de las medidas consideradas

\begin{tabular}{|r|c|c|c|}
\hline MEDIDAS & AGUA SUMINISTRADA (m3) & PRECIO (€/m3) & COSTE AGUA (€) \\
\hline TRASVASE & $39.602 .400,00$ & 0,5 & $19.801 .200,00$ \\
\hline e1 & $12.212 .620,00$ & 0,5 & $6.106 .310,00$ \\
\hline e3 & $43.000 .000,00$ & 0,4 & $17.200 .000,00$ \\
\hline \multicolumn{5}{|r|}{ e5 } & $38.814 .215,00$ & 0,68 & $26.393 .666,20$ \\
\hline CESIÓN DCHOS. & $69.601 .595,00$ & 0,68 & $47.329 .084,60$ \\
\hline e4 & $7.000 .000,00$ & 0,30 & $2.100 .000,00$ \\
\hline
\end{tabular}

Fuente: elaboración propia

e 1 análisis mostrado es del todo incompleto si no se consideran los costes que para el regante suponen la financiación de las medidas, pues tal y como la propia DMa establece, el principio de recuperación de los costes de los servicios del agua debe estar presente en las actuaciones del Plan. en el cuadro 7 se muestran la estimación del coste final por metro cúbico para cada una de las medidas. Para el caso del trasvase el coste se ha estimado en función del canon de regulación del trasvase, los costes de explotación (energía y bombeos; reparaciones y mantenimiento del sistema de suministro; gastos de personal y administración) y la amortización anual. Para el caso de la cesión de derechos se ha tenido en cuenta el canon del trasvase y el precio percibido por la Comunidad de $\mathrm{r}$ egantes por la cesión de su concesión. Por último para el caso de la desaladora el coste de agua se ha establecido en base a los estimados en el estudio de viabilidad de la misma. en el cuadro 7 se muestran el coste del agua de cada medida para el regante. Se ha estimado por otra parte que la productividad media del agua en la comunidad de regantes Cuevas de a lmanzora es de $0,47 € / \mathrm{m}^{3}$, lo que implica que el coste del agua para las medidas propuesta está en el límite de la viabilidad, si se considera la disposición a pagar por el regante que vendrá definida por la rentabilidad media de los cultivos más frecuentes en la zona regable. e n este caso no se ha tenido en cuenta la incertidumbre en los precios de los productos obtenidos, factor a considerar dada la creciente volatilidad de éstos en los últimos años.

\section{CONCLUSIONES}

1 a aplicación empírica que este trabajo propone muestra que el a Ce es una herramienta que permite hacer una valoración objetiva sobre el pool de medidas propuestas para alcanzar los objetivos ambientales de una masa de agua. No obstante hay que decir que este enfoque resulta incompleto pues no toma en consideración los costes sociales y ambientales de cada medida así como los beneficios que para la sociedad representa la recuperación del acuífero. Desde este punto de vista el análisis Coste-Beneficio está más ajustado a los principios que encierra la DMa en cuanto a la importancia de la consecución de los objetivos ambientales y sus implicaciones sociales frente a la simple valoración económica de las medidas como herramienta de decisión. 
en cualquier caso, el a Ce aplicado a las medidas que se proponen en este análisis, muestra que las medidas más favorables considerando el contexto socioeconómico en el que éstas se desarrollan, son aquellas que producen un mayor volumen de recurso al menor coste posible. 1 as medidas que conducen a esta situación son sobre todo las cesiones de derechos, ya que por los mismos costes que soportan los usuarios para el mantenimiento de las infraestructuras del trasvase, se abre la posibilidad de nuevos caudales disponibles, una vez hayan sido adquiridos sus derechos de uso. este hecho constata la importancia de considerar las medidas básicas en los planes de cuenca en las que se incluirían tanto la posibilidad de implementar cesiones como la aplicación de mecanismo de control que impida la continuación de los procesos de extracción de aguas del acuífero. e ste modelo de gestión sería más eficiente si se llevara a cabo una flexibilización de las actuales normas y leyes que regulan estas transacciones. De esta forma, las cesiones de derechos serán capaces de gestionar de una manera más eficaz y flexible los riesgos asociados a un contexto de incertidumbre en la disposición de recursos hídricos. 1 os resultados obtenidos en este trabajo, muestran la importancia de valorar las medidas en conjunto teniendo en cuenta las sinergias que presenta y los distintos escenarios posibles de incertidumbre en los resultados esperados. e n este caso la afirmación realizada sobre la idoneidad desde un punto de vista financiero no es correcta si no se complementa con un análisis de los efectos de las cesiones en terceras partes, que en este caso sería la zona de influencia del área de riego cedente de derechos y sobre todo los efectos ambientales sobre el sistema hídrico afectado, que paradójicamente se centra en la pérdida de caudales en la cabecera de la cuenca del Guadalquivir a pesar de que los usuarios directamente afectado se ubiquen en el curso medio y bajo de la cuenca. e s por ello que sería necesario incorporar a los costes del agua pagados por los regantes un canon complementario con el que se financien las compensaciones de los afectados por el trasvase y las cesiones, así como una tasa ambiental que cubriera los costes ambientales reparables.

No obstante y como futura línea de investigación que abre este análisis, se plantea el análisis de las medidas conducentes a la resolución de los problemas de contaminación de la masa de agua, pues esos persistirán aunque el acuífero deje de estar sobreexplotado. ante este grave problema ambiental y la dificultad de disponer de agua, es también importante plantearse una reflexión sobre el futuro de la agricultura en estas zonas a partir de un análisis riguroso de los costes desproporcionados. en esta línea es fundamental considerar que el carácter de excepcionalidad a la hora de alcanzar los objetivos ambientales que la DMa establece, debe ir asociado con algún tipo de contraprestación económica que de algún modo grave está excepción y repercuta en otras zonas a proteger. Por otra parte sería necesario realizar un análisis coste beneficio que permita contrastar el coste de las mismas con la valoración que la sociedad realiza sobre la consecución de los objetivos en forma de monetarización de los beneficios ambientales que la recuperación reporta. 


\section{REFERENCIAS BIBLIOGRÁFICAS}

aCUaMeD (2006); Informe de viabilidad de la desaladora en el Bajo almanzora, aguas de las Cuencas Mediterráneas S.a., Madrid, Marzo de 2006.

a Ge NCIa a NDa 1 Uza Del a GUa (2008); e studio de la Demarcación Mediterránea andaluza. repercusiones de la actividad humana en el estado de las aguas, Conserjería de Medioambiente de la Junta de andalucía, Sevilla, 2008.

aGeNCIa aNDalUza Del aGUa (2009); esquema de temas importantes de la Demarcación Mediterránea andaluza (eTI), Conserjería de Medioambiente de la Junta de andalucía, Sevilla, 2009.

alMaSrI, N.M. (2007); Nitrate contamination of groundwater: a conceptual management framework en: Environmental Impact assessment Review. Vol. 27, 220-242, 2007.

Ál Varez-F arIzO, B., HaNleY , N., BarBerÁN, r. Y lÁzarO, a. (2006); Choice Modelling at the 'Market Stall': Individual versus Collective Interest in environmental Valuation en: Ecological Economics, Vol. 60 14pp. 2006

Ál Varez-F arlzO, B. Y HaNleY , N. (2006); Improving the Process of Valuing Non-Market Benefits: Combining Citizens Juries with Choice Modelling, en: Land Economics, vol. 82, $\mathrm{n}^{\mathrm{o}}$ 3, 465-478, 2006.

a r r OJO, P., (coord.) (2001); e 1 Plan Hidrológico Nacional a debate, Bakeaz/Fundación Nueva Cultura del agua, Bilbao, 2001

BerBel, J., VIaGGI, D., MaNOS, B. (2008); estimating demand for irrigation water in european Mediterranean countries through MCDM models, en: Water Policy Journal, 2008.

BerBel, J., MeSa, P. Y Mar TíN Or TeGa, J. (2009); el análisis coste eficacia de los programas de medidas, en: la economía del agua de riego en españa, 5575, Gómez-limón y otros (eds.), Fundación Cajamar, almería, 2009.

BrOUWer, r. Y GeOrGIOU, S. (2007); economic Valuation of environmental and resources Costs and Benefits of Water Uses and Services in Water Framework Directive: Technical Guidelines for Practitioners, eU 6th FP aquaMoney Project: Development and Testing of Practical Guidelines for the assessment of environmental and resource Costs and Benefits in the WFD: www.aquamoney.org, 2007.

Cala Tra Va, J. Y GóMez-raMOS a. (2009); el papel de los mercados de agua como instrumento de asignación de recursos hídricos en el regadío español, en: la economía del agua de riego en españa, 295-319, Gómez-limón y otros (eds.), Fundación Cajamar, almería, 2009.

CIS (2003); Common Implementation Strategy for the Water Framework Directive (2000/60/eC), Guidance Document No 1 economics and the environment - The Implementation Challenge of the Water Framework Directive, Office for Official Publications of the european Communities, luxembour g, 2003.

COMMISSION eUrOPeaN (2003), economics and the environment: The implementation Challenge of the Water Framework Directive. a guidande Document. Bruselas, 2003. 
COr Te S, r . Ga r CÍa , r Y MOl INa , J. (2002); Claves para entender el modelo económico almeriense basado en la agricultura de alto rendimiento, en: Cajamar (eds.), 1 a agricultura mediterránea del siglo XXI, Fundación Cajamar, a lmería, 2002.

Del Saz, S., HerNÁNDez, F. Y Sala, r. (2009); estimación del valor económico de la calidad del agua de un rio mediante una doble aproximación: Una aplicación de los principios económicos de la Directiva Marco del agua, en: economía agraria y de los recursos naturales, Vol. 9, nº $1,2009$.

e STe Va N, a . Y Na r e DO, J. M. (2004); Ideas y propuestas para una nueva política del agua en e spaña, Bakeaz/Fundación Nueva Cultura del a gua, Bilbao, 2004.

GarrIDO, a. Mar TíNez-SaNT OS, P Y llaMaS r. (2006); Groundwater irrigation and its implications for water policy in semiarid countries: the Spanish experience, en: Hydrogeology Journal Vol. 14, 340-349, Springer Berlin / Heidelberg (eds.), 2006.

Ferrer, G. Y la rOCa, F. (2006); el papel de la economía en el desarrollo e implementación de la Directiva Marco del agua. ambigüedad conceptual y problemas prácticos, Universidad de Valencia, Mimeo, 2006.

GóMez, C.M. (2006); los costes y beneficios ambientales del proceso de decisión de la DMa, Hydronomic Convention, Barcelona 13-14 Noviembre, 2006.

GóMez-raMOS a. Y OlMeDIlla S. (2011); Valoración económica de las medidas destinadas a la recuperación de una masa de agua muy modificada. el caso del acuífero Bajo-almanzora (almería), en: revista de economía agraria y recursos naturales (en prensa).

KraNz, N., GÖrlaCH, B. Y INeTer WIeS, e. (2004); Making the right choices a methodology for selecting Cost effective measures for the WFD, en: Journal for european and Planning law , 1(3), 228-233, 2004.

la rOCa, F y G. Ferrer (2007); la economía en el desarrollo de la Directiva Marco del agua. Observatorio medioambiental Vol. 10 179-198.

llaMaS, r. Y Mar TÍNez-SaNT OS, P. (2005); Intensive Groundwater Use: Silent revolution and Potential Source of Social Conflicts, en: Journal of Water $r$ esources Planning and Management / Vol. $131 \mathrm{~N}^{\circ}$ 5, a SCe, (September/October), 337-341, 2005.

llaMaS, M.r. Y GarrIDO, a. (2007); lessons from intensive use in Spain: economic and social benefits and conflicts, en: The agricultural Groundwater revolution: Opportunities and Threats to Development, Giordano y Villholth (eds.), 266-295, CaB International, Wallingford, UK, 2007.

Mar TIN-Or TeGa, J. Y BerBel, J. (2008); Beneficios ambientales de la Directiva marco del agua: Conceptos y estimación, en: revista de estudios Geográficos, 1XIX (265), 609-635, 2008.

MarITINez-aller, J., MUNDa G. Y O'NeIll, J. (1998); Weak comparability of value as a fundation for ecological economics, en: ecological economics 26 , 277-286, 1998.

PalaNCar, M (2001); la nueva cultura del agua y el Plan hidrológico nacional. revista de Obras Públicas, Julio agosto 2001. No 3412. 47-49 
SÁNCHez PICóN, a., azNar SÁNCHez, J.a., azNar Pérez, P. (2009); el trasvase Tajo-Segura en la provincia de almería. evolución e integración de la oferta de recursos hídricos del Valle del almanzora, Caja de ahorros del Mediterráneo, almería, 2009.

SaHUQUIllO, a, CUSTODIO, e. Y llaMaS, r. (2009); la gestión de las aguas subterráneas, en: Tecnología del agua Vol. 305, 60-67, 2009.

TOlóN, a. Y laSTra, X. (2010); la agricultura intensiva del poniente almeriense. Diagnóstico e instrumentos de gestión ambiental, en: revista electrónica de Medio ambiente 2010, Vol. 8, 18-40, 2010.

\section{PUBLICACIONES (RELACIONADAS CON LA TEMÁTICA DEL ARTÍCULO)}

GóMez raMOS, a. y OlMeDIlla Pérez, S. (2011) Valoración económica de las medidas destinadas a la recuperación de una masa de agua muy modificada. el caso del acuífero Bajo-almanzora (almeria). revista de economía agraria y recursos Naturales (en prensa)

GIl, M, a. GarrIDO y a. GóMez-raMOS (2010), "Disentangling the social, macro and micro-economic effects of agricultural droughts: an application to Spanish irrigated agriculture" Options Méditerranéennes Serie a. Vol 95 Página inicial: 149 final:159 ISSN1016-121-X

GIl, M, a. GarrIDO y a. GóMez-raMOS (2010), How to link agricultural productivity, water availability and water demand in a risk context: a model to manage hydrological risks. Spanish Journal of Agricultural Research Vol 8, ISSN 1695-971-X. (2010) Special Issue (S2). Pp: 207-220.

GIl, M, a. GarrIDO y a. GóMez-raMOS (2010), economic analysis of drought risks: an application to irrigated agriculture in Spain, Agricultural Water ;anagement vol 98(5) páginas: inicial 823 final: 833. Marzo 2011

MaezTU, J. a. GóMez-raMOS y S. SÁNCHez. Mercados de agua: un instrumento novedoso en la gestión pública del agua. ambienta Volumen: 79 Páginas, inicial: 6 final:13. 2008.

MaezTU, J, GóMez-raMOS, a. GarrIDO, a. y SÁNCHez TaMarIT , S (2008). Instruments de mercat i gestió de léscassetat hídrica. DCIDOB Volumen: primavera 2008 Páginas, inicial 35 final: 40Fecha: 2008 Cidodb edicions Barcelona.

GóMez-lIMóN， J.a. y a. GóMez-raMOS (2008) Opinión pública sobre la agricultura del regadío en Castilla y león. Tierras de Castilla y león. Volumen: 142Páginas, inicial 120 final: 136 2008. Gestora de comunicaciones de Castilla y león (Valladolid)

GóMez-lIMóN, J.a. y a. GóMez-raMOS (2007), Opinión pública sobre la multifuncionalidad del regadío: el caso de Castilla y león. economía agraria y recursos Naturales Volumen: 7 (13).Páginas, inicial 3 final: 25, 2007. Mundi prensa (Madrid). 
IGleSIaS, e., a. GarrIDO y a. GóMez-raMOS. economics drought management index to evaluate water institutions' performance under uncertainty. The Australian Journal of Agricultural and Resources Economics. Volumen: 51, Páginas, inicial1 final:38 2007.

GóMez raMOS, a. y a. GarrIDO COlMeNerO , la gestión conjunta de los recursos del embalse de 'el Pintado' para fines agrícolas y de abastecimiento (Sevilla) mediante un contrato de opción. Ingeniería Civil Volumen: 139 (3), Páginas inicial: 125final: 132.2005

GóMez raMOS, a. y a. GarrIDO, Formal risk sharing mechanisms to allocate uncertain water resources: the case of option contracts. Water Resource Research Volumen: 40 (12), Páginas, inicial: 1 final: 112004

GóMez-raMOS, a, y a. GarrIDO COlMeNerO. la cesión de derechos de agua de la agricultura a los usos urbanos. Una aproximación a un contrato de cesión entre la z.r. del Viar y la Ciudad de Sevilla. andalucía Geográfica. Volumen: 10 Páginas, inicial: 55 final: 61.2004.

IGleSIaS, e. a. Garrido y a. GóMez. evaluation of drought in irrigated areas. Agricultural Economics. Volumen:29, Páginas, inicial: 211 final: 229 . 2003

GóMez raMOS, a., e. IGleSIaS Mar TINNez, ya. GarrIDO COlMeNerO. evaluación de la garantía de suministro de agua a la agricultura. Una aplicación a la cuenca del Guadalquivir. Ingenieria del agua. Volumen: 9 (3), Páginas, inicial: 279 final: 309. 2002

GIaNSaNTe, C. BaBIaNO, M., M. aGUIlar, a. GarrIDO, e. a. GóMezraMOS, e. IGleSIaS W. IISe and 1. MOral, Institutional adaptation to changing risk of water scarcity in the lower Guadalquivir Basin. Natural Resources Journal. Volumen: 42(3) Páginas, inicial: 521-final: 563. 2002

GarrIDO, a., e. IGleSIaS y a. GóMez raMOS. el agua subterránea en la gestión de las sequía. el impacto económico de la sequía en la agricultura. revista de la real academia de las Ciencias exactas Físicas y Naturales Volumen: 94(2), Páginas, inicial: 233 final: 245. 2000. 